\title{
ORIGINAL ARTICLE Individualised and health-related quality of life of persons with spinal cord injury
}

\author{
F Tramonti ${ }^{1}$, A Gerini ${ }^{2}$ and G Stampacchia ${ }^{2}$
}

\begin{abstract}
Study design: Cross sectional.
Objectives: To examine associations among functional status, health-related and individualised quality of life (QoL) and coping style in subjects with spinal cord injury (SCI).

Setting: Italy.

Methods: Forty subjects attending our hospital-based rehabilitation centre for SCI were administered the Spinal Cord Independence Measure-version III (SCIM-III), the Short Form-36 (SF-36), the Schedule for the Evaluation of Individual QoL-Direct Weighting (SEIQoL-DW) and the Coping Inventory for Stressful Situation (CISS) to assess functional status, health-related QoL, individualised QoL and coping strategies, respectively.

Results: The SCIM-III scores were positively correlated $(P<0.01)$ with the physical functioning subscale of the SF-36. The only scale that correlated $(P<0.01)$ with the SEIQoL-DW scores was the SF-36 mental health subscale. Positive correlations among taskoriented or avoidance-oriented coping and specific measures of health-related QoL emerged.

Conclusions: Data from the present study showed only a few associations among different measures of QoL and suggest that patientcentered evaluations of QoL are not necessarily or strictly related to functional status or health-related QoL. Coping strategies seem to be significantly related to the subjects perceptions of QoL, but mostly in the health-related domains.
\end{abstract}

Spinal Cord (2014) 52, 231-235; doi:10.1038/sc.2013.156; published online 17 December 2013

Keywords: spinal cord injury; quality of life; functional status; coping style

\section{INTRODUCTION}

The World Health Organisation defines quality of life (QoL) as 'an individual's perception of his/her position in life in the context of the culture and value systems in which he/she lives, and in relation to his/ her goals, expectations, standards and concern.' ${ }^{1}$ According to this definition, QoL is a complex concept, entailing the person's physical health and level of independence, as well as psychological well-being, social participation and the relationship with specific and relevant aspects of the environment. ${ }^{1,2}$ As a result of the complexity of this construct, it is not surprising that a large number of instruments measuring QoL have been developed in the field of medicine and health psychology, which often address different aspects and domains. ${ }^{2}$ Some of these instruments look at QoL globally, whereas others assess more specific domains. Conceptualisations and operationalisations of QoL can also be grouped on the basis of the extent to which health and physical well-being, or personal values are taken into account. In this respect, we can recognise two main approaches for the study of QoL in medical and psychological settings: one focused on health-related measures and the other concentrated on individualised measures, which are also referred to as patient-centered evaluations. ${ }^{3}$ These perspectives should not be viewed as incompatible, given that the domains they investigate are often complementary and relevant. On one hand, individualised QoL measures give us information on the level of satisfaction of aspects that are specifically relevant for the subjects, which are otherwise inaccessible. Conversely, health-related measures can shed light on the effects of health on specific life domains. Furthermore, evaluating different aspects of QoL also has important implications in terms of health care policies, since it can be helpful in identifying different dimensions of health and well-being that could be used to inform interventions.

The importance of proper QoL assessment is vital for persons with spinal cord injury (SCI), as it is a condition where different degrees of physical limitations and related complications (for example, pain, bowel, bladder and sexual problems, and so on.) can cause significant restrictions in daily activities, health and well-being. ${ }^{4}$ In this respect, previous findings suggest a complex relationship between physical impairment and QoL in this population. For instance, measures that are strictly related to physical health and functioning typically find a significant difference between people with SCI and the general population, whereas instruments that evaluate subjects wider perspectives on life satisfaction do not often find large differences between these groups. ${ }^{2}$ One possible reason for this discrepancy is due to the relative importance of how health is viewed in relation to QoL by patient populations. In depth examinations of instruments measuring individualised QoL have found that health is not always listed as one of the most salient aspects of life satisfaction for subjects recruited in clinical settings. ${ }^{5}$ These findings confirm how QoL in disease/injury conditions seem to be a multifaceted construct, which is influenced by several factors. In particular, the interplay among

${ }^{1}$ Clinical Psychology Unit, Department of Neurosciences, Azienda Ospedaliero-Universitaria Pisana, Pisa, Italy and ${ }^{2}$ Centre for Spinal Cord Injuries, Department of Neurosciences, Azienda Ospedaliero-Universitaria Pisana, Pisa, Italy

Correspondence: Dr F Tramonti, Clinical Psychology Unit, Department of Neurosciences, Azienda Ospedaliero-Universitaria Pisana, Via Roma 67, Pisa 56127, Italy.

E-mail: tramonti.francesco@gmail.com

Received 20 September 2013; revised 23 October 2013; accepted 10 November 2013; published online 17 December 2013 
psychosocial variables and physical functioning might be playing a key role. ${ }^{6}$

There is evidence suggesting that coping strategies act as a potential mediator, together with social participation and support, between the physical consequences of SCI and the psychological adjustment. ${ }^{7,8}$ However, the results of studies investigating the role of coping in shaping psychological adjustment and QoL are not always in agreement. In some of such studies, coping styles have been identified as strong predictors of psychological adjustment to SCI, and active and problem-focused styles have been recognised as the most beneficial. ${ }^{9}$ In other works, the relevance of specific strategies or attitudes, such as social reliance, has been highlighted. ${ }^{10}$ On the other hand, recent reviews emphasise how in many other articles the evidence that some coping strategies or styles are more adaptive than others is not robust enough. ${ }^{11,12}$ Probably, the poor agreement among different studies can be partially explained by the differences in the outcome measures adopted, and evaluating the associations between coping and different measures of QoL might be of help in better understanding such differences.

Age and especially time since injury have often been found to be positively related to psychological adjustment, ${ }^{13}$ whereas the role of gender has received less attention and the available data suggest a weak impact of such a variable on life satisfaction. ${ }^{14}$ Given all these premises, the aims of the present study are to examine the relationships among functional status and different measures of QoL in a population of subjects with SCI, and to consider the role of coping style, age, time since injury and gender. Given the aforementioned discrepancies arising from different evaluations of QoL in subjects with SCI, and given the poor correlations of different QoL measures found in other patient populations, we can hypothesise that few associations among such measures will occur in our study population as well. The outcome of the present study could provide a better understanding of the relationship between individualised and health-related QoL, which are both necessary approaches for conducting a proper evaluation of the possible benefits arising from rehabilitation and other treatment options for patients with SCI. Furthermore, the results could also contribute to the understanding of the role of coping and other variables, such as age, time since injury and gender, which is not completely understood.

\section{MATERIALS AND METHODS}

A sample of 40 subjects with SCI (Table 1) was derived from the evaluation of 52 patients who consecutively attended our hospital-based centre for SCI from December 2011 to September 2012. Seven subjects who had been injured within the six months before our testing were excluded, whereas three refused to participate and two did not complete all the tests. None of the subjects had concurrent traumatic brain injuries, which were also considered as exclusion criteria for their possible impact on cognitive functioning. Twenty-eight subjects were married, whereas twelve lived with their family of origin, or alone but in proximity to parents or close relatives. Most of the subjects were living in towns or small cities in central Italy, with only two of them living in rural areas. Scales for the evaluation of functional status, health-related QoL, individualised QoL and coping style were administered to the sample via faceto-face interviews, which are all included as part of the standard clinical care at our centre. All the applicable institutional and governmental regulations concerning the ethical use of human subjects were followed during the course of this research.

Functional status was measured by the Spinal Cord Independence Measureversion III (SCIM-III), a disease-specific scale assessing levels of autonomy in a broad range of physical activities and functions. ${ }^{15}$ Health-related QoL was measured with the Short Form-36 (SF-36), a widely used instrument consisting of eight subscales that identify different domains of health status: physical functioning, physical role functioning, bodily pain, general health perceptions,
Table 1 Study population characteristics

Characteristics

\section{Current age}

Mean and s.d.

54.25

12.96

Median and range

54

23-77

Years since injury

Mean and s.d.

Median and range

Gender

Male

$n=28$

$70 \%$

Female

$n=12$

$30 \%$

Traumatic/non-traumatic injuries

Traumatic

$n=18$

$45 \%$

Non-Traumatic

$n=22$

$55 \%$

Paraplegialtetraplegia

Paraplegia

$n=28$

$70 \%$

Tetraplegia

$n=12$

$30 \%$

Neurological category

Ventilator dependent

C1-C4 AIS A, B, C

$n=0$

C5-C8 AIS A, B, C

$n=1$

$2.5 \%$

T1-sS5 AIS A, B,

$22.5 \%$

All AIS D

$n=9$

$n=17$

$n=13$

$42.5 \%$

$32.5 \%$

Abbreviation: s.d, standard deviation.

vitality, social role functioning, emotional role functioning and mental health. ${ }^{16}$ Although some criticism has been made about the appropriateness of certain items, especially those referring to 'walking', the SF-36 has been validated in various studies on SCI populations and is generally considered as a reliable instrument for the evaluation of health-related QoL in this population. $^{2}$

Individualised QoL was assessed with the Schedule for the Evaluation of Individual QoL-Direct Weighting (SEIQoL-DW), a semi-structured interview that evaluates the importance of aspects of life chosen by the subject and the actual satisfaction from such aspects. ${ }^{17}$ As a result of its validity and usefulness in assessing reprioritisation issues and individual perceptions of QoL, the schedule is extensively used in QoL research in medical and psychological settings. ${ }^{18}$ With respect to SCI literature, the SEIQoL-DW has only been administered in few studies, where it provided helpful insights into the complex relationship between functional status and life satisfaction. ${ }^{19,20}$ For the assessment of coping style, the Coping Inventory for Stressful Situation (CISS) was employed. The CISS is a valid and reliable questionnaire based on the distinction of three different tendencies in facing adversities: task-oriented coping, emotion-oriented coping and avoidance-oriented coping. ${ }^{21}$ Taskoriented strategies are those focused on active problem-solving, whereas the emotion-oriented style includes venting, focusing on affective reactions or even self blame. The avoidance-oriented coping subscale covers different strategies of distraction, such as trying not to think too much about problems and social diversion. Descriptive statistics and frequencies were calculated for all data. The correlations among the scales and subscales were calculated by means of the Spearman's $R$, and the Kruskal-Wallis test was used for the comparison between men and women.

\section{RESULTS}

The mean scores of QoL and functional status measures of the sample are reported in Table 2. When compared with normative data, the 
Table 2 Mean scores of QoL and functional status measures with standard deviations

\begin{tabular}{|c|c|c|c|c|c|c|c|c|c|c|}
\hline & SEIQOL & $\begin{array}{c}\text { Physical } \\
\text { functioning }\end{array}$ & $\begin{array}{l}\text { Physical role } \\
\text { functioning }\end{array}$ & Bodily pain & $\begin{array}{c}\text { General health } \\
\text { perceptions }\end{array}$ & Vitality & $\begin{array}{c}\text { Social } \\
\text { functioning }\end{array}$ & $\begin{array}{c}\text { Emotional } \\
\text { role } \\
\text { functioning }\end{array}$ & $\begin{array}{l}\text { Mental } \\
\text { health }\end{array}$ & SCIM \\
\hline $\begin{array}{l}\text { General } \\
\text { sample }\end{array}$ & $69.85 \pm 18.84$ & $17.00 \pm 18.18$ & $25.00 \pm 35.80$ & $60.80 \pm 30.59$ & $49.67 \pm 22.43$ & $57.62 \pm 16.37$ & $64.37 \pm 27.08$ & $60.00 \pm 43.50$ & $69.15 \pm 15.21$ & $56.32 \pm 19.92$ \\
\hline Women & $70.77 \pm 21.03$ & $25.42 \pm 20.83$ & $14.58 \pm 29.11$ & $55.42 \pm 34.67$ & $42.33 \pm 18.17$ & $57.08 \pm 16.85$ & $63.54 \pm 33.90$ & $58.33 \pm 51.49$ & $70.17 \pm 18.63$ & $60.00 \pm 24.77$ \\
\hline Men & $69.46 \pm 18.22$ & $13,39 \pm 15,99$ & $29,46 \pm 37,92$ & $63,11 \pm 29,05$ & $52,82 \pm 23,62$ & $57,86 \pm 16,47$ & $64,73 \pm 24,31$ & $60,71 \pm 40,63$ & $68,71 \pm 13,86$ & $54,75 \pm 17,74$ \\
\hline
\end{tabular}

Abbreviation: SEIQoL, Schedule for the Evaluation of Individual Quality of Life.

Table 3 Most representative SEIQoL-DW domains and average importance and satisfaction ratings

\begin{tabular}{lcc}
\hline Domains & $\begin{array}{c}\text { Average estimated } \\
\text { importance (\%) }\end{array}$ & $\begin{array}{c}\text { Average satisfaction } \\
\text { ratings (0-100) }\end{array}$ \\
\hline Couple/family relationships & $52.08 \pm 10.82$ & $74.50 \pm 12.80$ \\
Physical health/functional independence & $30.12 \pm 9.70$ & $42.24 \pm 16.28$ \\
Work/leisure activities & $28.94 \pm 10.50$ & $45.48 \pm 20.14$ \\
\hline
\end{tabular}

SF-36 subscale scores that seem to reveal a stronger impact of SCI are those related to the physical domains, especially for the physical functioning and physical role functioning subscales. Examining the contents that emerged from the SEIQoL-DW interviews, the aspects of life that were most frequently chosen and highly rated in terms of importance, can be grouped into three main domains: physical health/functional independence, couple/family relationships and topics related to social participation, leisure activities and work (Table 3).

No significant correlation between the SEIQoL-DW and SCIM-III scores emerged, and the SF-36 subscales showed only few correlations with the aforementioned measures. A positive correlation $(P<0.01$; $1-\beta=0.99)$ was found with SF-36 physical functioning subscale and SCIM-III scores. A positive correlation $(P<0.01 ; 1-\beta=0.63)$ was also detected between the SEIQoL-DW and SF-36 mental health subscale (Table 4).

With regards to coping style (CISS), a positive correlation $(P<0.01 ; 1-\beta=0.64)$ between task-oriented coping and avoidanceoriented coping was found; the latter positively correlated with mental health $(P<0.01 ; 1-\beta=0.64)$, general health perceptions $(P<0.05$; $1-\beta=0.65)$, emotional role functioning $(P<0.05 ; 1-\beta=0.65)$ and vitality $(P<0.05 ; 1-\beta=0.64)$, and negatively $(P<0.01 ; 1-\beta=0.76)$ with physical functioning and the SCIM-III scores. Task-oriented coping positively correlated $(P<0.05 ; 1-\beta=0.64)$ with physical role functioning and social role functioning.

Emotion-oriented coping showed negative correlations with bodily pain $(P<0.01 ; 1-\beta=0.64)$, social role functioning $(P<0.01$; $1-\beta=0.63)$, emotional role functioning $(P<0.01 ; 1-\beta=0.67)$ and mental health $(P<0.01 ; 1-\beta=0.64)$. Age negatively correlated $(P<0.05 ; 1-\beta=0.65)$ with physical functioning and with the SCIM-III scores (Table 3 ). Time since injury correlated positively $(P<0.05 ; 1-\beta=0.65)$ with task-oriented coping, whereas no gender differences emerged on any of the measures. Consecutively, in order to deepen the investigation of functional status, comparisons between subjects with tetraplegia and paraplegia, as well as between subjects with complete and incomplete injuries were also performed, but in both the cases no significant difference emerged.

\section{DISCUSSION}

Despite small sample size suggesting caution in drawing definitive conclusions from the results, our data suggest that the different measures of QoL show only few associations between each other in this study population. Only the mental health subscale of the SF-36 correlated with the SEIQoL-DW scores. With regards to impairment, the only correlation that emerged was between the SCIM-III and the physical functioning subscale of the SF-36. These results are consistent with previous findings in the literature, ${ }^{2,4}$ and confirm the need for a complex approach in the study of QoL, which can frame all the relevant aspects of this construct, and that can take into account the relationships among different domains. A plausible inference we can draw from these findings is that the pattern of positive correlations found among functional status, health-related and individualised QoL might be described on a continuum as visually pictured in Figure 1.

Health-related QoL seems to cover a vast area whose boundaries intertwine with those of functional status and individualised QoL, and create areas of intersection. In other words, the more we consider the psychological aspects of QoL, the closer we come to individualised QoL, whereas the measures of health-related QoL that are strictly related to physical impairment seem to be associated with functional status measures. Similarly, when compared with the norms, the physical domains of health-related QoL seem to be much more affected than the psychological ones. This confirms the relevance of psychosocial factors in the perception of life satisfaction and in the adjustment to physical impairment. In this respect, assuming that disability involves health conditions and contextual factors, both personal and environmental, it is not surprising that physical impairment could not alone shape the perception of QoL. It should also be noted that the sample we studied consisted of subjects who were not in an acute phase of SCI, a phase that usually poses different psychological demands and during which it is plausible that physical impairment is a more relevant aspect and a stronger determinant of life satisfaction. When entering the chronic phase of the condition, subjects who positively react to the onset of physical impairment typically redefine priorities in their lives. This process, especially for people with SCI, helps them to face the constraints of the physical condition without forgoing an active role in their families and in society. ${ }^{13}$

The role of coping strategies can also be seen from this developmental point of view. Our finding that emotion-oriented coping negatively correlated with various dimensions of QoL confirms the assumption that these kinds of strategies, despite being normative and to some extent helpful in the acute phase of SCI, can be dysfunctional in the long run. In the chronic phase, in fact, more task-oriented strategies are needed for the reestablishment of realistic and satisfactory goals throughout the life cycle. The finding that avoidanceoriented coping positively correlated with several dimensions of QoL could be interpreted in the light of the construction of the CISS 
Table 4 Correlations

\begin{tabular}{|c|c|c|c|c|c|c|c|c|c|c|c|c|c|c|c|}
\hline & CISS Task & CISS Emotion & CISS Avoidance & SEIQoL & PF & $R P$ & $B P$ & $G H$ & $V T$ & SF & $R E$ & $M H$ & $S C I M$ & Age & $T S I$ \\
\hline CISS - T & 1.00 & - & - & - & - & - & - & - & - & - & - & - & - & - & - \\
\hline CISS - E & -0.16 & 1.00 & - & - & - & - & - & - & - & - & - & - & - & - & - \\
\hline CISS - A & $0.45^{* *}$ & -0.25 & 1.00 & - & - & - & - & - & - & - & - & - & - & - & - \\
\hline SEIQoL & 0.26 & -0.21 & 0.30 & 1.00 & - & - & - & - & - & - & - & - & - & - & - \\
\hline PF & 0.00 & 0.10 & $-0.42^{* *}$ & -0.11 & 1.00 & - & - & - & - & - & - & - & - & - & - \\
\hline $\mathrm{RP}$ & $0.36^{*}$ & -0.21 & 0.17 & 0.07 & 0.09 & 1.00 & - & - & - & - & - & - & - & - & - \\
\hline $\mathrm{BP}$ & 0.07 & $-0.42^{* *}$ & 0.04 & 0.14 & 0.06 & 0.29 & 1.00 & - & - & - & - & - & - & - & - \\
\hline $\mathrm{GH}$ & 0.15 & -0.22 & $0.33^{*}$ & 0.28 & 0.02 & $0.36^{*}$ & $0.47^{* *}$ & 1.00 & - & - & - & - & - & - & - \\
\hline VT & 0.22 & -0.20 & $0.37^{*}$ & 0.28 & -0.02 & 0.29 & $0.43^{* *}$ & $0.58^{* *}$ & 1.00 & - & - & - & - & - & - \\
\hline SF & $0.38^{*}$ & $-0.45^{* *}$ & 0.17 & 0.07 & 0.16 & 0.25 & $0.37^{*}$ & 0.25 & $0.34^{*}$ & 1.00 & - & - & - & - & - \\
\hline RE & 0.29 & $-0.51^{* *}$ & $0.36 *$ & 0.18 & 0.03 & $0.54 * *$ & $0.35^{*}$ & 0.24 & 0.15 & $0.47^{* *}$ & 1.00 & - & - & - & - \\
\hline $\mathrm{MH}$ & 0.17 & $-0.46^{* *}$ & $0.45^{* *}$ & $0.41^{* *}$ & 0.01 & $0.34^{*}$ & $0.49 * *$ & $0.58^{* *}$ & $0.65^{* *}$ & $0.43^{* *}$ & $0.46^{* *}$ & 1.00 & - & - & - \\
\hline SCIM & -0.06 & 0.30 & $-0.54 * *$ & -0.11 & $0.72^{* *}$ & 0.10 & -0.01 & -0.00 & -0.21 & -0.05 & -0.06 & -0.25 & 1.00 & - & - \\
\hline Age & -0.00 & -0.06 & 0.12 & 0.13 & $-0.32^{*}$ & -0.21 & -0.09 & -0.16 & 0.18 & 0.01 & -0.13 & 0.04 & $-0.35^{*}$ & 1.00 & - \\
\hline TSI & $0.32 *$ & -0.05 & 0.13 & -0.11 & 0.12 & 0.29 & -0.00 & -0.21 & -0.15 & 0.07 & 0.18 & 0.01 & 0.04 & -0.21 & 1.00 \\
\hline
\end{tabular}

Abbreviations: BP, bodily pain; CISS, coping inventory for stressful situation; GH, general health perceptions; $\mathrm{MH}$, mental health; PF, physical functioning; RP, physical role functioning; $\mathrm{RE}$, emotional role functioning; SF, social role functioning; TSI, time since injury; VT, vitality.

${ }^{*} P<0.05$.

$* * P<0.01$

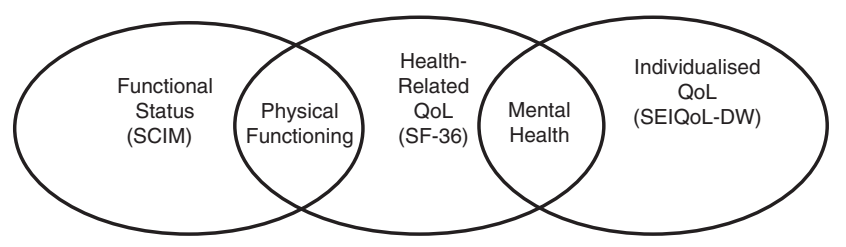

Figure 1 Pattern of associations among different measures of QoL and functional status.

questionnaire, where the avoidance subscale is built upon items referring to distraction and social diversion, rather than to denial. Interestingly, avoidance-oriented coping is negatively correlated with physical functioning, both in relation to the SCIM-III scores and to the physical functioning subscale of the SF-36, a finding that could suggest that such strategies might be particularly meaningful for those subjects who have a greater degree of impairment. In general, the CISS scores correlated only with the health-related measures of QoL, whereas no correlation with the SEIQoL-DW scores emerged. This is not surprising, as coping strategies are surely important variables in psychological adjustment but it is unlikely that they alone can shape the overall perception of QoL. Appraisal, personal or cultural beliefs and environmental variables, such as the quality of family relationships, access to social networks and the presence of social barriers, likely have a key role too. ${ }^{22,23}$ This is indeed confirmed by the frequent occurrence of these domains as relevant aspects chosen by our study population during the SEIQoL-DW administration. Despite lamenting a relevant impact of social and environmental barriers on their activities, when interviewed our subjects appeared quite satisfied, on average, with their life context and intimate relationships. More in general, it has to be noted that previous research into coping strategies of subjects with SCI has typically used, as outcome measures, psychological distress, physical well-being or health-related QoL, rather than individualised QoL.

Age seems to be negatively associated with physical functioning but not to the QoL measures that are related to psychological domains. This infers that positive adjustment to injury shows some kind of stability, despite the physical difficulties of aging with SCI. ${ }^{24}$ Possibly, such difficulties could be counterbalanced by processes of adjustment strengthened by age and experience, as confirmed by the improvement of QoL or by the decrease of depressive symptoms over time, which are well documented in SCI studies. ${ }^{13,14}$ If we consider time since injury, our data supports the hypothesis that a longer duration of SCI is significantly associated with a higher prevalence of task-oriented coping strategies, confirming the increasing relevance of such adaptive strategies over time. Further research, and especially longitudinal studies, should be conducted in line to increase our knowledge of these important aspects. Doing so will enable us to account for improvements in medical treatment for SCI, and for the increasing number of people aging with SCI. Longitudinal studies would be of help also in deepening our understanding of the role of coping, as correlations found in crosssectional studies allow only to infer hypotheses about the associations between attitude or behaviour and markers of life satisfaction, without providing evidence of causal relationships. Further investigation is also needed to better understand the role of gender. Although our findings revealed no differences, this might be attributable to our sample being relatively small and primarily male.

In conclusion, our findings confirm the complexity of the QoL construct and seem to support the evidence that different scales could measure aspects or investigate domains that are not necessarily or strictly related to one another. This might also suggest the importance of a more extensive use of individualised instruments, like the SEIQoL-DW, in conditions like SCI, where they can be of help in identifying relevant aspects of QoL that could be underestimated or not included in health-related measures. More in general, the few associations between health-related and individualised evaluations, together with the significant role of coping, testify to the relevance of psychological factors in shaping life satisfaction. This confirms the importance of psychological counselling, in addition to rehabilitation programmes and social interventions, as a treatment option that could be of precious help in the adjustment to SCI and in preventing the transition from physical impairment to actual disability.

\section{DATA ARCHIVING}

There were no data to deposit. 


\section{CONFLICT OF INTEREST}

The authors declare no conflict of interest.

1 The WHOQoL Group. Protocol for New Centres. WHO, 1994

2 Hill MR, Noonan VK, Sakakibara BM, Miller WC, and the SCIRE Research Team. Quality of life instruments and definitions in individuals with spinal cord injury: a systematic review. Spinal Cord 2010; 48: 438-450.

3 Carr AJ, Higginson IJ. Measuring quality of life: are quality of life measures patient centred? BMJ 2001; 322: 1357-1360.

4 Whalley Hammel K. Quality of life after spinal cord injury: a meta-synthesis of qualitative findings. Spinal Cord 2007; 45: 124-139.

5 McGee H. Quality of life. In Kaptein A and Weinman J (eds). Health Psychology. Blackwell: Malden, 2004, pp 234-257.

6 Mathew KM, Ravichandran G, May K, Morsley K. The biopsychosocial model and spinal cord injury. Spinal Cord 2001; 39: 644-649.

7 Galvin LR, Godfrey HPD. The impact of coping on emotional adjustment to spinal cord injury (SCl): review of the literature and application of a stress appraisal and coping formulation. Spinal Cord 2001; 39: 615-627.

8 Elfstrom M, Ryden A, Kreuter M, Taft C, Sullivan M. Relations between coping strategies and health-related quality of life in patients with spinal cord lesion. J Rehabi Med 2005; 37: 9-16.

9 Bonanno GA, Kennedy P, Galatzer-Levy IR, Lude P, Elfström ML. Trajectories of resilience, depression and anxiety following spinal cord injury. Rehabil Psychol 2012; 57: 236-247.

10 Kennedy P, Lude P, Elfström ML, Smithson EF. Psychological contributions to functional independence: a longitudinal investigation of spinal cord injury rehabilitation. Arch Phys Med Rehabil 2011; 92: 597-602.

11 Post MWM, van Leeuwen CMC. Psychosocial issues in spinal cord injury: a review. Spinal Cord 2012; 50: 382-389.
12 van Leeuwen CM, Kraaijeveld S, Lindeman E, Post MW. Associations between psychological factors and quality of life ratings in persons with spinal cord injury: a systematic review. Spinal Cord 2012; 50: 174-187.

13 Krause JS. Aging and life adjustment after spinal cord injury. Spinal Cord 1998; 36: 320-328.

14 Dijkers MPJM. Correlates of life satisfaction among persons with spinal cord injury. Arch Phys Med Rehabil 1999; 80: 867-876.

15 Itzkovich M, Gelernter I, Biering-Sorensen F, Weeks C, Laramee MT, Craven BC et al. The Spinal Cord Independence Measure (SCIM) version III: reliability and validity in a multi-center international study. Disabil Rehabil 2007; 29: 1926-1933.

16 Ware JE, Sherbourne CD. The MOS 36-item short-form health survey (SF-36). Conceptual framework and item selection. Med Care 1992; 30: 473-483.

17 O'Boyle CA, McGee HM, Hickey A, Joyce CRB, Browne J, O'Malley K et al. The Schedule for the Evaluation of Individual Quality of Life (SEIQoL). Administration Manual. Royal College of Surgeons in Ireland: Dublin, 1993.

18 Wettergren L, Kettis-Lindblad A, Sprangers M, Ring L. The use, feasibility and psychometric properties of an individualised quality-of-life instrument: a systematic review of the SEIQoL-DW. Qual Life Res 2009; 18: 737-746.

19 Effing TW, van Meeteren NL, van Asbeck FW, Prevo AJ. Body weight-supported treadmill training in chronic incomplete spinal cord injury: a pilot study evaluating functional health status and quality of life. Spinal Cord 2006; 44: 287-296.

20 Levack P, Graham J, Kidd J. Listen to the patient: quality of life of patients with recently diagnosed malignant cord compression in relation to their disability. Palliat Med 2004; 18: 594-601.

21 Endler NS, Parker JDA. Assessment of multidimensional coping: Task, emotion, and avoidance strategies. Psychol Assess 1994; 6: 50-60.

22 Kennedy P, Evans M, Sandhu N. Psychological adjustment to spinal cord injury: the contribution of coping, hope and cognitive appraisals. Psychol Health Med 2009; 14: 17-33.

23 Chevalier Z, Kennedy P, Sherlock O. Spinal cord injury, coping and psychological adjustment: a literature review. Spinal Cord 2009; 47: 778-782.

24 Thompson L, Yakura J. Aging related functional changes in persons with spinal cord injury. Top Spinal Cord Inj Rehabil 2006; 6: 69-82. 